\title{
An Enhanced Indoor Positioning Method Based on Wi-Fi RSS Fingerprinting
}

\author{
Marwan Alfakih, and Mokhtar Keche
}

\begin{abstract}
In WiFi-based indoor positioning, the received signal strength (RSS) measurements are commonly used to estimate the mobile user location. However, these measurements significantly fluctuate over time and are susceptible to human movement, multipath and Non-Lineof-Sight (NLOS) propagation, which reduce the location accuracy. In this paper, an enhanced positioning method based on the nearest neighbor algorithm is proposed. The set of the RSS samples recorded from several Access Points (APs) is used rather than their average, for reducing the location errors introduced by the RSS variations and the multipath problem. The proposed algorithm, named the Nearest $K^{\text {th }}$ Nearest Neighbor (NK-NN) is experimentally evaluated and compared to other powerful methods. The results show that the proposed method outperforms these methods.
\end{abstract}

Index Terms - Indoor localization, nearest neighbor, received signal strength, Wi-Fi fingerprinting, wireless communication.

\section{INTRODUCTION}

$\mathrm{O}$ VER the last decades, researchers have developed various approaches for mobile positioning to meet the growing demand for accurate positioning systems. Achieving a good positioning, the Global Positioning System [1] (GPS) has been widely used in outdoor environments. However, the GPS is unavailable inside buildings, due to the weakness of its signal that cannot penetrate obstacles. Several alternative technologies have been used to locate a Mobile User (MU) in indoor environments, such as ultrasonic, infrared, pedestrian inertial, and radio frequency (Wi-Fi, RFID, Bluetooth, Ultra Wide Band (UWB), and Zigbee) technologies [2-6].

$\mathrm{Wi}-\mathrm{Fi}$ is the most popular technology used for indoor locali-

Manusript received September 4, 2018; revised December 14, 2018. Date of publication January 28, 2019. The associate editor Prof. Nikola Rožić has been coordinating the review of this manuscript and approved it for publication.

M. Alfakih (corresponding author) and M. Keche are with the Laboratoire Signaux et Images, Département d'Electronique, Université des Sciences et de la Technologie d'Oran, Mohamed Boudiaf, (USTO, MB), BP.1505 EL M'nouer, Oran, 31000, Algérie (e-mails: marwan.alfakih@univ-usto.dz, m_keche@yahoo.com).

Digital Object Identifier (DOI): 10.24138/jcomss.v15i1.612. zation, due to its availability in most buildings and the widespread of its signal over long distances.

However, the WiFi-based location techniques suffer from the problem of signal fluctuations, caused by the interferences from other devices operating in the same band and multipath propagation. Many Wi-Fi based methods have been proposed to address these effects, using path loss model [7]. However, the use of these methods is restricted, due to the requirement of map information [8] and a fixed receiver's direction [9]. On the other hand, the location fingerprinting [10] is considered as the most viable approach for indoor localization; it uses a radio map for matching the spatial positions and the signal strength recorded at these positions. This technique consists of two principal phases: a training phase and a locating phase. During the training phase, the RSS samples are collected at each Reference Point (RP) and stored in a training database (fingerprints). The locating phase compares these measurements with the actual measurement, recorded at an unknown location, to find the similar fingerprint and return the corresponding location as the estimated location. However, this approach suffers from the problems resulting from noisy RSS values and similar fingerprints.

This paper presents a fingerprinting method using the Wi-Fi strength signal for indoor positioning. An enhanced version of the nearest neighbor algorithm, called the NK-NN, is proposed to reduce the positioning errors, produced by multipath and RSS variations. We use the set of the RSS samples, i.e. all the RSS samples, collected for training and testing, instead of their averages, which are usually used in the standard KNN and its variants. To screen out the noisy RSS testing samples, each testing sample is compared to each fingerprint and only the sample that has the minimum distance is selected for the position's calculation. Then, a classification process is performed on the $\mathrm{K}^{\text {th }}$-nearest training samples of different RPs. This process helps in pruning the noisy training RSSs and excluding them from the localization. The experimental results show that the NK-NN method performs better than other similar methods.

The rest of the paper is organized as follows. Section 2 reviews related work on location fingerprinting using RSS; it is followed by the description of the proposed positioning method 
in Section 3. The performance of the proposed method is evaluated experimentally and compared to those of other positioning methods in Section 4. Finally, Section 5 draws some conclusions.

\section{LOCATION FINGERPRINTING BASED ON RSS MEASUREMENTS}

In the specialized literature, location fingerprinting based on RSS measurements is classified into two main categories: deterministic and probabilistic techniques. In this section, a review of the main location fingerprinting techniques is presented.

\section{A. Deterministic Techniques}

Deterministic techniques rely mainly on using the average of the training RSS measurements and comparing them to the actual RSS measurement, to find the similar fingerprint. The $\mathrm{KNN}$ is the basic localization fingerprinting method, which was first used in the Radar system [11]. It first finds the K-nearest RPs of the mobile user location, based on the Euclidian distance between the training and testing RSS measurements. Then the coordinates of these $\mathrm{K}$ nearest points are averaged to find the position of the mobile user. In [12], the location accuracy is improved by using the weighted average of the coordinates of the $k$ closest points; the weights are calculated by inverting the Euclidean distances. The experimental results in [12] show that the WKNN can achieve a better accuracy than the KNN and the simple NN methods. In [13], we have proposed an improved method, called Improved Nearest Neighbor (INN), based on the NN technique. This method uses all the training RSS samples without averaging, and chooses as position of the mobile user the position that has the highest occurrence among $\mathrm{M}$ positions, estimated using the NN algorithm. However, its drawback is that the results depend on the storage order of the training samples in the MxL training matrix, where $\mathrm{L}$ is the number of reference points and $\mathrm{M}$ is the number training samples per reference point.

In the previous methods, a fixed number $k$ of nearest neighbors is used. However, this number may be changed during the positioning process to improve the positioning accuracy. Using this idea, Shin et al. [14] proposed the Enhanced Weighted KNN (EWKNN) algorithm, which improves the accuracy by changing dynamically the number $k$. Therein, the Euclidian distance is calculated and compared to a given threshold, and the RPs that have a distance greater than this threshold are eliminated. Then, the distances of the remaining RPs are sorted in ascending order and the differences between the smallest and the other distances are calculated. Only, the RPs whose difference is less than or equal to the average of all distances are retained in the location calculation. According to the experimental results in [14], this method outperforms the NN, the KNN and the WKNN methods.

Petric et al [15] proposed another location method to determine dynamically an optimal value of the number of the nearest neighbors, $k$, based on analyzing the radio map and the environment structure. This algorithm is referred to as Dynamic KNN (DKNN). Therein, after finding the first (closest) neighbor in radio domain, it is decided to include the following nearest neighbors based on their distance from the first one in radio and space domains. If the first point is in a distance larger than a threshold, the number of neighbors, $\mathrm{k}$, is set to a large value $K_{\max }$ and the $K_{\max }$ nearest points are used to calculate the MU's position. Otherwise, the next neighbors should be analyzed. The DKNN reduces the maximum errors by $35 \%$ compared to the standard KNN method [14].

\section{B. Probabilistic Techniques}

Instead of representing the location fingerprints by the mean of the RSS samples as in the nearest neighbor approach, the distribution of the these samples are used in the probabilistic approach, which models the location fingerprint with the conditional probability and utilizes the Bayesian inference concept for location estimation $[5,16]$. The probabilistic approach allows for a better handling of the noise in wireless channels. The location estimation is based on the likelihood or posterior probabilities. In particular, given the fingerprints data, the probability of the measurement conditionally to different locations is calculated and the location with the highest probability is selected as the estimated location.

The posterior distribution of the location conditionally to the measurement, denoted $x$, can be calculated using the Bayes theorem:

$$
p(l \mid x)=\frac{p(x \mid l) p(l)}{p(x)}=\frac{p(x \mid l) p(l)}{\sum_{i=1}^{M} p\left(x \mid l_{i}\right) p\left(l_{i}\right)},
$$

where $p(x \mid l)$ is the likelihood function, $p(l)$ is the prior probability of location $l, p(x)$ is a normalizing constant that does not depend on the location, and $M$ is the number of RPs in the area of interest. If the prior is uniform, the likelihood function completely determines the posterior distribution of the location. Several methods can be used for estimating this likelihood function, including the Kernel [10, 16], the histogram [17] and the Gaussian mixture [18] methods. It can be shown that the estimate that minimizes the mean squared errors is given by:

$$
E(l \mid x)=\sum_{i=1}^{M} l_{i} p\left(l_{i} \mid x\right),
$$

where $l_{i}$ is the location of the ith reference point. Including the coordinates of each reference point in the location estimation can reduce the large-sized errors [16].

The Kernel method is a probabilistic based method used to estimate the likelihood function. It approximates the probability density function of an observation (measurement) $x$ at a location $l$ by using the Gaussian function: 


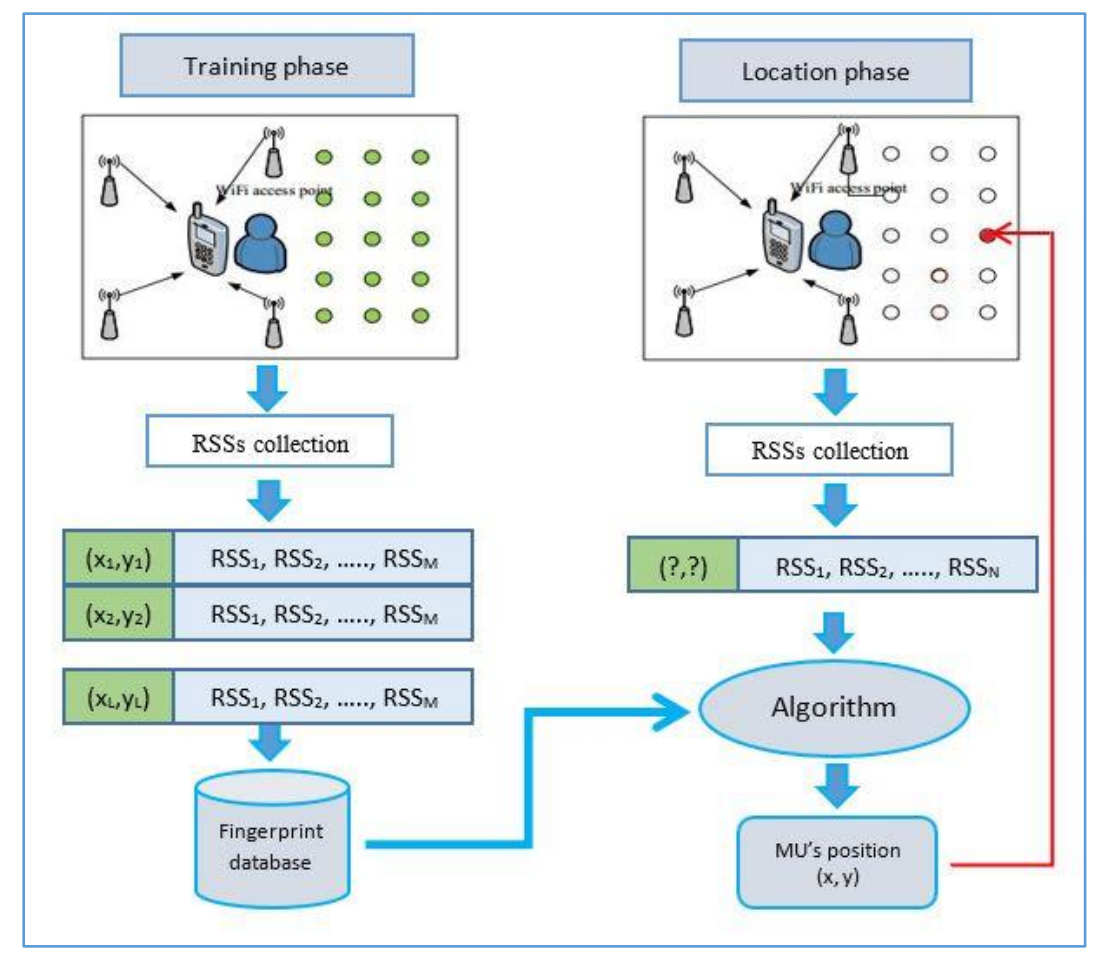

Fig. 1. Methodology of the proposed method.

$$
p(x \mid l)=\frac{1}{n} \sum_{i=1}^{n} \frac{1}{\sqrt{2 \pi} \sigma} e^{-\frac{\left(x-x_{i, l}\right)^{2}}{2 \sigma^{2}}},
$$

where $n$ is the number of training RSS data in each location, $x_{i, l}$ is the ith RSS training sample at location 1 , and $\sigma$ is an adjustable parameter that determines the Kernel width.

The likelihood function may also be estimated using the histogram method. During the training phase, for each location $l_{i}$, a histogram is constructed from the RSSs measurements acquired at this location. At the testing phase, the algorithm retrieves the probability of the testing RSS sample conditional to a location, from the histogram at this location. The number of bins used to compute the histogram is a parameter that must be chosen to obtain the best results.

\section{The Proposed NeAREST K ${ }^{\mathrm{TH}}$ NeAREST NeIGHBor (NK- NN) POSITIONING METHOD}

The nearest neighbor methods use the average of the RSS measurements, which reduces the computational time in the location process. However, they do not accommodate the existence of temporal variations and RSSs fluctuations. Furthermore, if the environmental condition has changed significantly from the training to the locating phase, the fingerprints require to be recollected to have an accurate positioning. The proposed method can mitigate these problems by using a large amount of the signal strength measurements during the training and location phases. The Euclidian distance is used to match the current measurements to those of the database. The proposed positioning method consists of two phases: the offline training phase and the online location phase, as shown in Fig. 1.

\section{A. The Offline Training Phase}

The training phase, also known as the planning phase, is dedicated to build the training database (fingerprints). This training database reflects how the signal propagates in the area of interest. The area of interest is divided into a grid in which each node represents a reference point. At each reference point, M RSS samples are collected from each access point, and stored in a database, together with their corresponding positions.

\section{B. The Online Location Phase}

In this phase, the user seeks to discover his whereabouts by collecting $N$ RSS samples from each access point at his current unknown location. Given these measurements, the algorithm searches in the fingerprinting database, constructed in the previous phase, to find the similar fingerprint, which is retained as the user estimated location. To achieve an efficient estimation, we propose an algorithm based on the nearest neighbor method. Our algorithm operates according to the following steps (Algorithm 1):

1) Each sample of the N RSS testing samples is compared to each sample of the M RSS training samples collected at each reference point, using the Euclidian distance, calculated as follows:

$$
D_{j, m}(l)=\sqrt{\sum_{i=1}^{n}\left(R S S_{j, i}^{\prime}-R S S_{m, i}(l)\right)^{2}},
$$

where $R S S_{j, i}^{\prime}$ is the $j^{\text {th }}$ RSS testing sample $(j=1, \ldots, N)$ collected from the $i^{\text {th }}$ access point $(i=1, \ldots, n)$ and $R S S_{m, i}(l)$ is the $m^{\text {th }}$ training sample $(m=1, \ldots, M)$ 


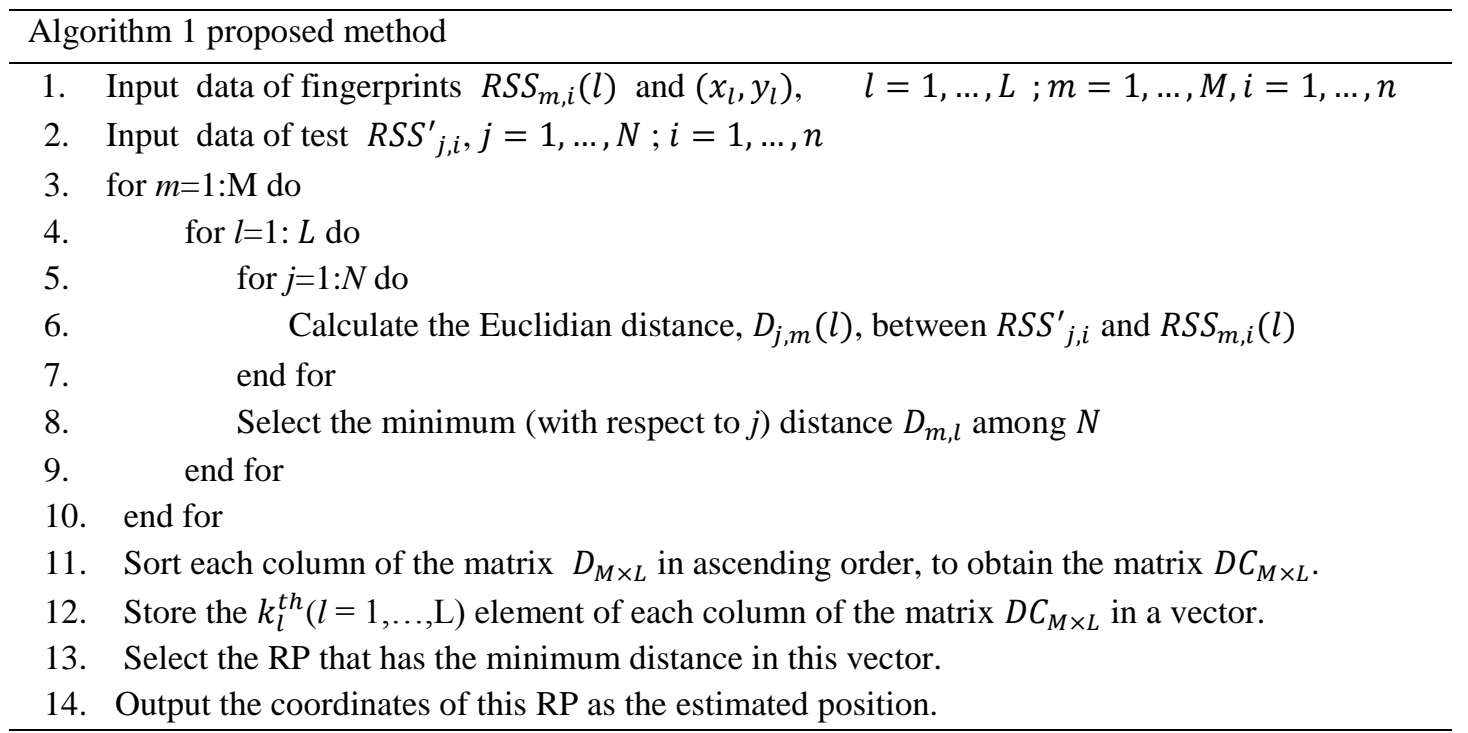

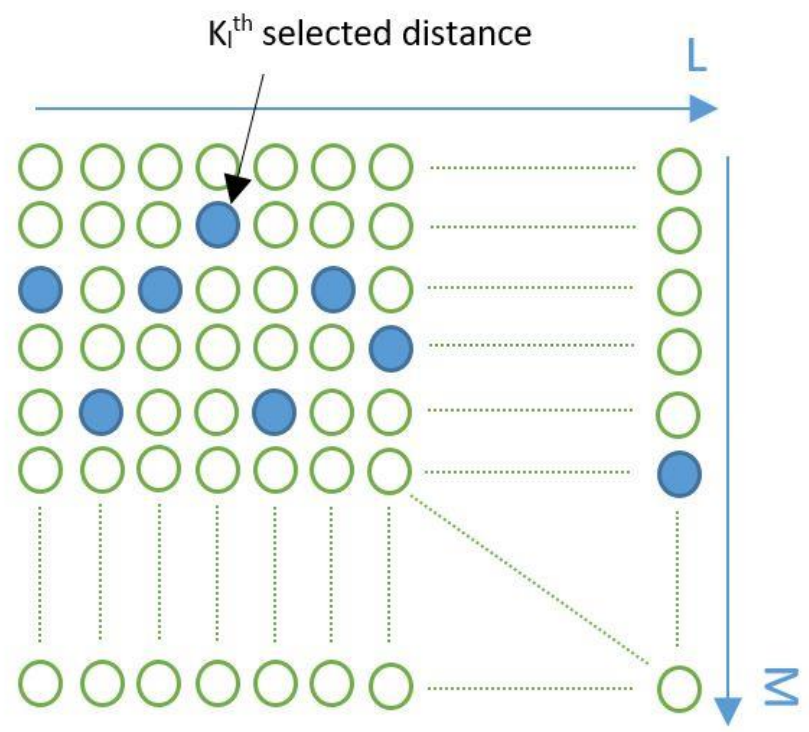

Fig. 2. Graphical representation for $k_{l}$ selection process.

recorded at the $l^{\text {th }}$ location (RP), from the $i^{\text {th }} \mathrm{AP}$, in the area of interest.

2) For each training sample, at each reference point, only the minimum distance among $N$ distances is selected for the following process. Consequently, $M \times L$ distances are obtained and stored in a matrix, $D_{M \times L}$, of dimension $M \times L$. This pruning allows discarding the noisy RSS testing samples collected during the location process.

3) Classify the distances in each column of the matrix $D_{M \times L}$ in ascending order to obtain a matrix $D C_{M \times L}$, so that $D C_{1, l}<$ $D C_{2, l}<\cdots<D C_{M, l}, l=1, \ldots, L$.

4) Store the $k_{l}^{\text {th }}$ element of each column of the matrix $D C_{M \times L}$ in a vector, i.e. take for the $\mathrm{l}^{\text {th }} \mathrm{RP}$ the $\mathrm{k}_{\mathrm{l}}^{\text {th }}$ smallest distance, as shown in Figure 2. The $\mathrm{k}_{\mathrm{l}}^{\text {th }}$ selected distance is indicated by the blue circle for each column of the matrix $D_{M \times L}$.

Notice that the RPs may be affected different values of the parameter $\mathrm{k}$, since they are not equally placed vis-à-vis of the access points. This means that some RPs are privileged compared to other RPs. The choice of the best value for $\mathrm{k}_{\mathrm{l}}, \mathrm{l}=$ $1, \ldots, \mathrm{L}$ is determined by trial and error using cross-validation

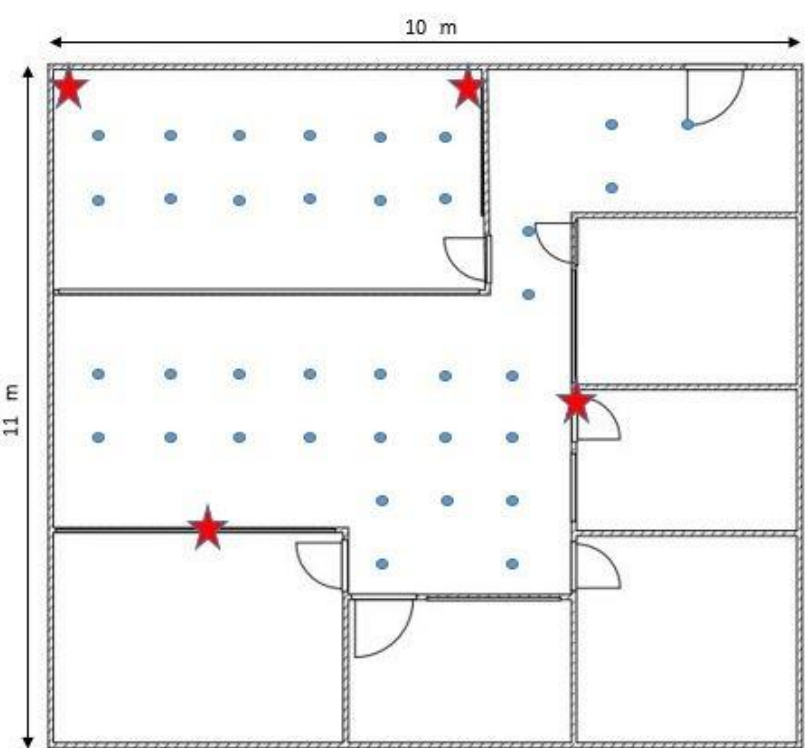

Fig. 3. Positions of the RPs and the APs in the Laboratory of Signals and Images.

with the training data. This data is divided in 10 folds, nine folds are used for training and the tenth fold for testing to determine by trial and errors the best value of the parameter $\mathrm{k}$ for each RP. Several values for the set $\mathrm{k}_{\mathrm{l}}, \mathrm{l}=1, \ldots, \mathrm{L}$ are tried and the set of values that provides the best generalization is selected.

5) Finally, from the constructed vector choose the location of the reference point that has the minimum distance as the estimated position of the user. Therefore, this algorithm could be named the nearest $\mathrm{k}^{\text {th }}$ nearest neighbor.

\section{EXPERIMENTAL RESULTS}

In this section, we describe the testbed's layout and the data collection, and discuss the obtained experimental results. The performance of the proposed method is compared to those of other methods available in literature. The effects of the number of training and testing RSS samples on the performance of the 


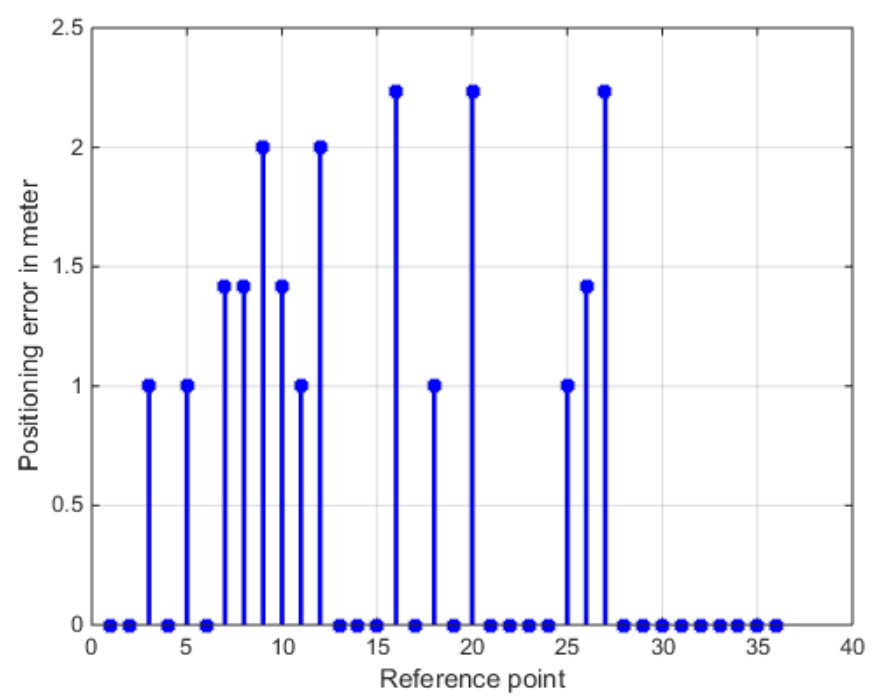

Fig. 4. Positioning errors of the NK-NN localization method at different RPs.

proposed method are also studied.

Our experiment was conducted in the Signal and Images Laboratory, situated on the third floor of the Electronics Department at the Sciences and Technology University of Oran. This testbed area of approximately $(11 \mathrm{~m} \times 10 \mathrm{~m})$ was covered using 4 TP-Link access points, as shown in Fig. 3. Thirty-six reference points, separated by $1 \mathrm{~m}$, were considered. The fingerprints were collected at these RPs and stored into the fingerprint database. We collected 1000 RSS samples, at a rate *of 20 samples/second, from each AP. This data represents the training data. The testing data was collected later in the same day in the same conditions and with the same hardware. At each reference point, 100 measurements were recorded. The mobile user is a Toshiba Laptop, running windows XP Service Packet 3, and equipped with a D-Link AirPlusG DWL-G650 Wi-Fi card.

A main issue in the proposed NK-NN algorithm is how to choose the best values of the indices $k_{l}$. These values were determined experimentally, using 10-folds cross-validation with the 1000 training samples. We could determine a set of values, which provides no localization errors, i.e. a perfect generalization. This set is : $k_{14}=k_{17}=k_{18}=250, k_{19}=$ $130, k_{32}=10, k_{34}=50$ and $k_{l}=95$, for the other values of $l$.

Figure 4 plots the positioning errors of the NK-NN algorithm versus the reference points, using the above set of $k_{l}$ and the testing data. It can be noticed that the NK-NN algorithm can locate the user's position exactly (positioning error $=$ zero) in about $61 \%$ of the reference points, while in $25 \%$ of the RPs the error is between $1 \mathrm{~m}$ and $1.4 \mathrm{~m}$, and in about $14 \%$ this error is between $2 \mathrm{~m}$ and $2.24 \mathrm{~m}$. The NK-NN method can locate the position of a mobile with a mean error of 0.59 meters.

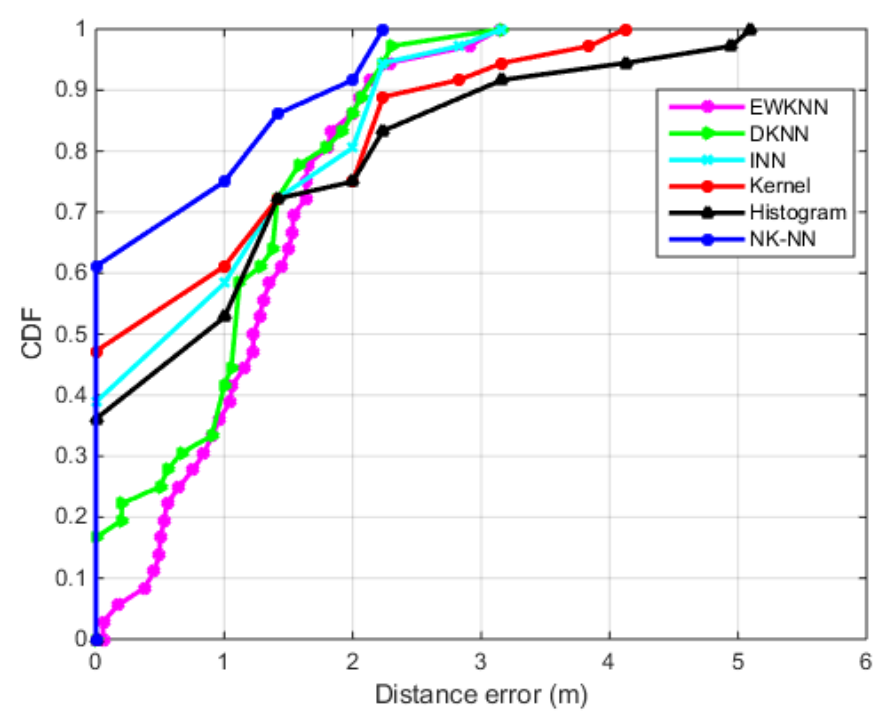

Fig. 5. CDF of the positioning errors of the NK-NN method compared to other methods.

TABLE I

Position ERrors' Statistics of DifFEREnt Positioning MEthods.

\begin{tabular}{lccccc}
\hline \multirow{2}{*}{ Method } & \multicolumn{5}{c}{ Distance Error (m) } \\
\cline { 2 - 6 } & Mean & Median & $67 \%$ & $90 \%$ & Max \\
\hline EWKNN & 1.28 & 1.25 & 1.53 & 2.08 & 3.14 \\
DKNN & 1.12 & 1.12 & 1.39 & 2.10 & 3.16 \\
INN & 1.04 & 1.00 & 1.26 & 2.16 & 3.16 \\
Kernel & 1.05 & 1.00 & 1.22 & 2.47 & 4.12 \\
Histogram & 1.34 & 1.00 & 1.30 & 2.98 & 5.10 \\
NK-NN & 0.59 & 0 & 0.42 & 1.82 & 2.24 \\
\hline \hline
\end{tabular}

The performance of the proposed NK-NN method is compared to that of other positioning methods in Fig. 5, where its Cumulative Density Functions (CDF) of the location errors, is plotted together with those of the EWKNN, the DKNN, the INN, the Kernel and the histogram methods. In $67 \%$ of cases, the NK-NN method can locate the mobile user's position within 0.42 meter, whereas the EWKNN, the DKNN, the INN, the Kernel and the histogram methods can locate this position within $1.53,1.39,1.26,1.22$ and 1.30 meters, respectively. Table 1 lists the statistics of the position errors (mean, median, $67 \%, 90 \%$ and maximum errors), for the six positioning methods. It can be noted that in terms of mean error, the NKNN method has a better performance than the other methods, with improvements of $53.91 \%, 47.32 \%, 43.25 \%, 43.81 \%$, and $55.97 \%$, over the EWKNN, the DKNN, the INN, the Kernel and the histogram methods, respectively.

In indoor localization, the acquired RSS measurements are usually affected by multipath, NLOS and human movement, which lead to reduced localization accuracy. The use of a large size of RSS measurements may limit the errors resulting from these impairments. Hence, we analyze in this paper the impacts 


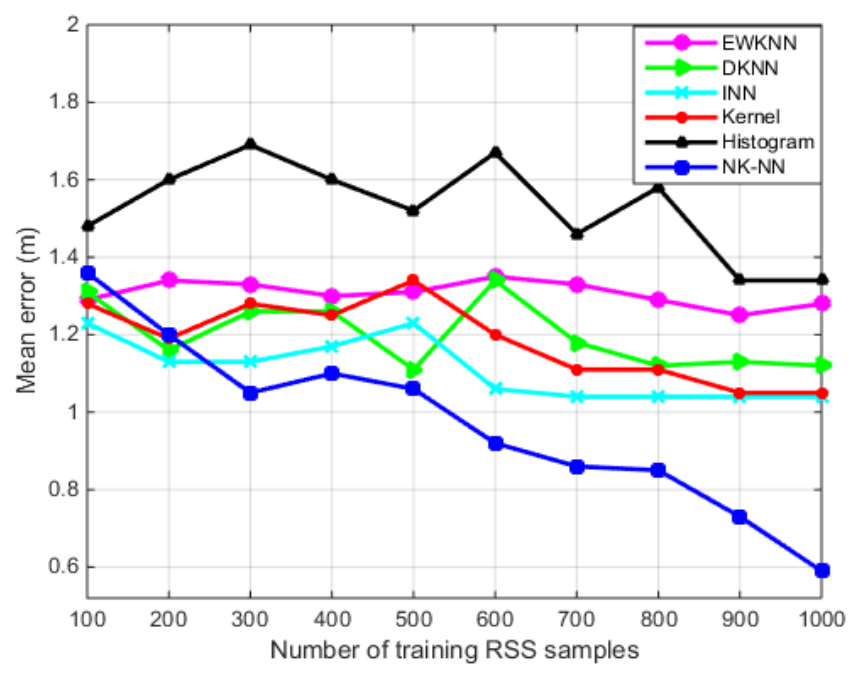

Fig. 6. Effect of the number of training samples on the accuracy of different positioning methods.

of the numbers of training and testing RSS samples on the location accuracy, for each of the above positioning methods. Figure 6 plots the mean localization error versus the number of training samples for each method. It can be noted that in the deterministic methods, the variations in the positioning errors in function of the number of training samples are small, because the average of the RSS measurements is used; in the probabilistic methods, these errors oscillate but overall tend to decrease when the number of samples increases, while in the proposed NK-NN method, they decrease when the number of samples increases, which means that the large number of samples is well exploited. From this figure, it can be observed that beyond 200 training samples, the NK-NN method has the best accuracy, compared to the other methods.

Figure 7 plots the mean localization error versus the number of testing samples, for the six positioning methods. It can be noted that in the five other methods, no decrease in the mean error occurs when the number of testing samples is increased beyond a certain value, which depends on the used method, whereas in the NK-NN method, the positioning error decreases when the number of testing samples increases. This can be explained by the fact that no averaging is performed in this proposed method. Hence, increasing the number of testing samples just helps to select the correct nearest RP by screening out the noisy RSS values.

From the above discussion, we conclude that increasing the numbers of the RSS samples used in the training and testing phase plays an important role for enhancing the location accuracy in the proposed method. However, the counterpart of this is an increase in the computational time.

To evaluate the computational complexity of the NK-NN algorithm and compare it to those of the other algorithms, we use the running time parameter. Table 2 lists the running times of the EWKNN, DKNN, INN, Kernel, histogram and NK-NN algorithms, which have been implemented using Matlab 2014,

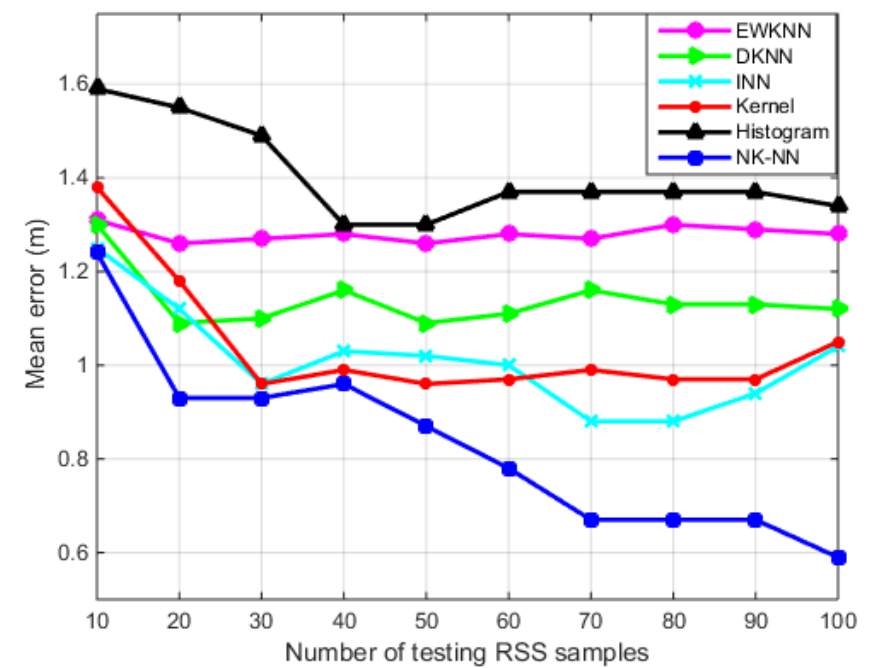

Fig. 7. Effect of the number of testing samples on the accuracy of different positioning methods.

TABLE II

RUNNING TIME OF DiFFERENT POSITIONING ALGORITHMS.

\begin{tabular}{cc}
\hline \hline Method & $\begin{array}{c}\text { Computational time } \\
\text { (Second) }\end{array}$ \\
\hline EWKNN & $3.31 \times 10^{-4}$ \\
DKNN & $3.78 \times 10^{-4}$ \\
INN & 0.79 \\
Kernel & 2.32 \\
Histogram & 0.08 \\
NK-NN & 0.62 \\
\hline \hline
\end{tabular}

running on a Dell Laptop, equipped with a processor Intel (R) Core (TM) i5-6200U CPU @ 2.30 GHz and 8 GB of RAM, and operated with windows 10, 64 bits. These running times were obtained using 1000 training samples and 100 testing samples. It is well known and this is confirmed by Table 2, that the deterministic methods that use the average of RSS samples, like the EWKNN, and the DKNN, have a lower complexity than those that use the distribution of the RSS samples, like the INN, the Kernel, the histogram, and the NK-NN. From this table, it can be observed that the NK-NN method takes 0.62 second to calculate the mobile user's position. This time is to be compared with 0.79 and 2.32 seconds, the time required by the INN and the Kernel methods, which are the closest methods, in terms of location accuracy, to the NK-NN method.

In the previous experiment, all the reference points were used for both training and testing. For a further assessment, the experiment is modified so that the reference points are divided into about $20 \%$ for testing and the other $80 \%$ for training, as shown in Figure 8. The reference points that are used for testing are indicated by green squares, while those used for training are in blue. Figure 9 plots the CDFs of the location errors for the EWKNN, the DKNN, the Kernel, the histogram, and the NKNN methods. As expected, it can be observed that the location accuracy decreases for all positioning methods, and the CDFs 


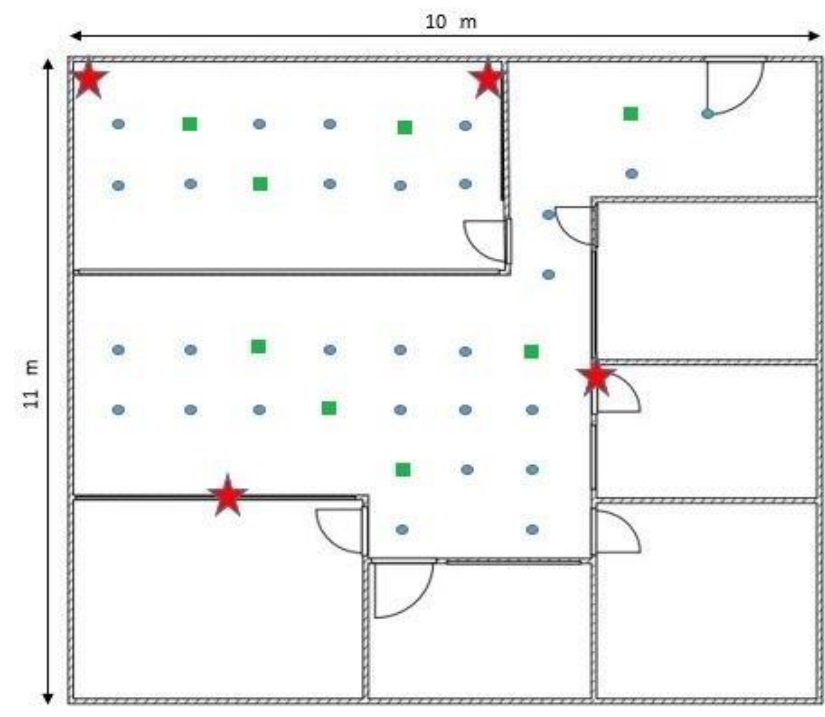

Fig. 8. Positions of the training RPs indicated by blue dotes and the testing RPs indicated by green squares.

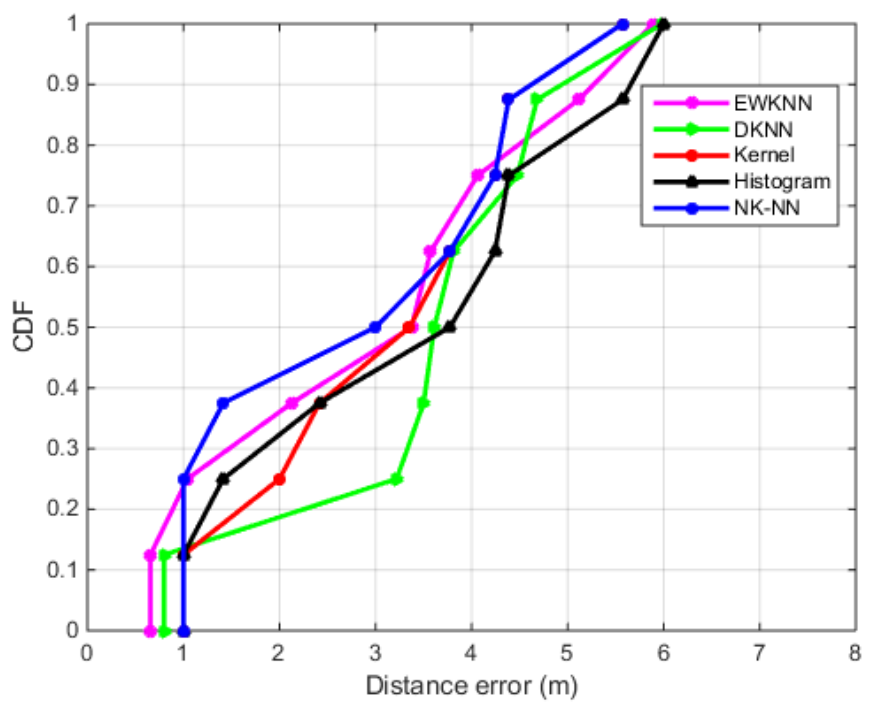

Fig. 9. CDF of the positioning errors of the NK-NN method compared to other methods.

do not began from zero, since the training and the testing points are separated by at least 1 meter. In this second experiment, it can be noted that in average, the NK-NN method can locate the mobile user's location within 3.05 meters, while the EWKNN, the DKNN, the Kernel and the histogram methods can locate this position within 3.23, 3.76, 3.34, and 3.60 meters, respectively, as reported in Table 3. Form Figure 9 and Table 3, it can be observed that the NK-NN method remains the best. If all reference points were used for both training and testing and in addition points in between these reference points were used for testing, which is the more realistic situation, the results would be in between those of the first and second experiments.

\section{CONCLUSION}

In this paper, an enhanced positioning method is presented to improve the location accuracy of indoor positioning, using the Wi-Fi signals. The set of the RSS samples is used rather than
TABLE III

Position ERrors' Statistics OF DifFERENT POSITIONING Methods.

\begin{tabular}{lccccc}
\hline \hline \multirow{2}{*}{ Method } & \multicolumn{5}{c}{ Distance Error $(\mathrm{m})$} \\
\cline { 2 - 6 } & Mean & Median & $67 \%$ & $90 \%$ & Max \\
\hline EWKNN & 3.23 & 3.47 & 3.75 & 5.27 & 5.89 \\
DKNN & 3.76 & 3.71 & 4.05 & 4.94 & 5.98 \\
Kernel & 3.34 & 3.56 & 3.94 & 4.62 & 5.57 \\
Histogram & 3.60 & 4.01 & 4.30 & 5.66 & 6.00 \\
NK-NN & 3.05 & 3.37 & 3.94 & 4.62 & 5.57 \\
\hline
\end{tabular}

their average, for reducing the errors produced by the noise in wireless channels. The presented experimental results show that the proposed method has the best performance, compared to other positioning methods from the specialized literature. By using the NK-NN method, the location accuracies of the EWKNN, the DKNN, the INN, the kernel, and the histogram methods are improved by 54\%, 47\%, 43\%, 44\%, and 56\%, respectively. The effects of the numbers of training and testing RSS samples on the performance of the proposed algorithm are also investigated.

\section{REFERENCES}

[1] R. Bajaj, S.L. Ranaweera, and D.P. Agrawal, "GPS location tracking technology," IEEE Computer, vol. 35, no. 4, pp. 92-94., 2002. doi:10.1109/MC.2002.993780

[2] O. Onalaja, M. Adjrad, and M. Ghavami, "Ultra-wideband-based multilateration technique for indoor localization," IET Communications, vol. 8, n.10, pp. 1800-1809, 2014. doi: 10.1049/iet-com.2013.0815

[3] Z. Zhang, Z. Lu, V. Saakian, X. Qin, Q. Chen, and L.R. Zheng, "Itemlevel indoor localization with passive UHF RFID based on tag interaction analysis," IEEE Transactions on Industrial Electronics, vol. 61, no. 4, pp. 2122-2135, 2014. doi: 10.1109/TIE.2013.2264785

[4] K. Kaemarungsi, R. Ranron, and P. Pongsoon, "Study of received signal strength indication in Zigbee location cluster for indoor localization," in Proceeding of the International Conference on Electrical Engineering/Electronics, Computer, Telecommunications and Information Technology, Thailand, May 2013, pp. 1-6. doi:10.1109/ECTICon.2013.6559612

[5] M. Youssef, A. Agrawala, and A. Shankar "WLAN location determination via clustering and probability distributions," in Proceeding of the first International Conference on Pervasive Computing and Communications, 2003, pp. 143-150. doi:10.1109/PERCOM.2003.1192736

[6] Y. Wang, X. Yang, Y. Zhao, Y. Liu, and L. Cuthbert, "Bluetooth positioning using RSSI and triangulation methods," in Proceeding of Consumer Communications and Networking Conference (CCNC), 2013, pp. 837-842. doi:10.1109/CCNC.2013.6488558

[7] K. El-Kafrawy, M. Youssef, A. El-Keyi, and A. Naguib, "Propagation modeling for accurate indoor WLAN RSS-based localization," in Proceeding of the International Conference on Vehicular Technology Fall, Ottawa, 2010, pp. 1-5. doi:10.1109/VETECF.2010.5594108.

[8] Z. Xiang, S. Song, J. Chen, H. Wang, J. Huang, and X. Gao, “A wireless LAN-based indoor positioning technology," IBM Journal of research and development, vol. 48 no. 5.6, pp.617-626, 2004. doi: 10.1147/rd.485.0617.

[9] K. Kaemarungsi, and P. Krishnamurthy "Properties of indoor received signal strength for WLAN location fingerprinting," in Proceeding of the First Annual International Conference on Mobile and Ubiquitous 
Systems: Networking and Services, 2004, pp. 14-23. doi:10.1109/MOBIQ.2004.1331706

[10] A. Kushki, K.N. Plataniotis, and A.N. Venetsanopoulos, "Kernel-based positioning in wireless local area networks," IEEE Transactions on Mobile Computing, vol. 6, no.6, pp. 689-705, 2007.

[11] P. Bahl, and V.N. Padmanabhan, "Radar: An in-building RF-based user location and tracking system," in Proceeding of the 19th Annual Joint Conference on Computer and Communications Societies, 2000, vol. 2, pp. 775-784. doi: 10.1109/INFCOM.2000.832252

[12] B. Li, J. Salter, A.G. Dempster, and C. Rizos, "Indoor positioning techniques based on wireless LAN," in Proceeding of the International Conference on Wireless Broadband and Ultra Wideband Communications, 2006, pp. 13-16. http://hdl.handle.net/10453/19580

[13] M. Alfakih, M. Keche, and H. Benoudnine, "An improved nearest neighbors (INN) algorithm for accurate WIFI based indoor positioning," European Navigation Conference (ENC), 2015, Bordeaux, France.

[14] B. Shin, J.H. Lee, T. Lee, and H.S. Kim, "Enhanced weighted k-nearest neighbor algorithm for indoor Wi-Fi positioning systems," in Proceeding of the 8th International Conference on Computing Technology and Information Management, Korea (South), 2012, pp. 574 - 577. https://ieeexplore.ieee.org/abstract/document/6268565/

[15] M. Petric, A. Neskovic, and N. Neskovic, "Dynamic k nearest neighbours model for mobile user indoor positioning," in the 23rd Telecommunications Forum Telfor (TELFOR), 2015, pp. 165-168. doi:10.1109/TELFOR.2015.7377439

[16] T. Roos, P. Myllymaki, H. Tirri, P. Misikangas, and J. Sievänen, “A probabilistic approach to WLAN user location estimation," International Journal of Wireless Information Networks, vol. 9, no.3, pp. 155-164, 2002. https://link.springer.com/article/10.1023/A:1016003126882

[17] M. Youssef, and A. Agrawala, "The Horus WLAN location determination system," in Proceeding of the 3rd International Conference on Mobile Systems, Applications, and Services, Seattle, Washington, 2005, pp. 205218. doi:10.1145/1067170.1067193

[18] M. Alfakih, M. Keche, and H. Benoudnine, "Gaussian mixture modeling for indoor positioning WIFI systems," in Proceeding of the 3rd International Conference on Control Engineering and Information Technology (CEIT), Tlemcen, Algeria, May 2015, pp. 1-5. doi:10.1109/CEIT.2015.7233072

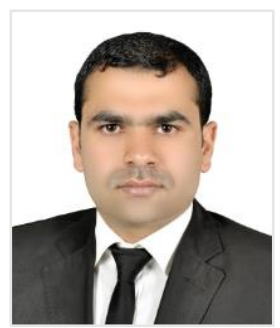

Marwan Alfakih received his B.S. and M.S. in Communication Technics (2010) and Signal Processing Applied to Communications (2012), respectively, from the University of Science and Technology of Oran, Mohamed Boudiaf, (USTO, MB) Oran, Algeria. He is currently pursuing the Ph.D. degree in Modern Technics of Information and Communications at the Laboratory of signals and images, Electronics Department, University of Science and Technology, Mohamed Boudiaf, (USTO, MB), Oran, Algeria. His research interests are related to Applied Signal Processing, Indoor Positioning, Tracking, Navigation and Wireless Communications.

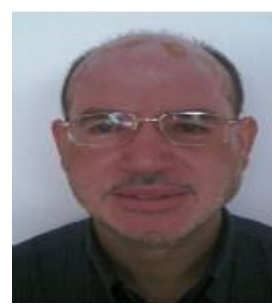

Mokhtar Keche received the Engineering degree in Telecommunications from the Ecole Nationale Superieure des Telecommunications (ENST), Paris, France in 1978, and the Doctor of Engineering degree and $\mathrm{PhD}$ from the University of Rennes in France and the University of Nottingham in UK in 1982 and 1998, respectively. He is Professor at the Electronic Department of University of Sciences and Technology, Mohamed Boudiaf, (USTO, MB), Oran, Algeria. His research interests are in the area of Digital Communication, Array Processing, Multi Target Tracking, and Road Traffic Congestion Estimation. 